23

\title{
Сравнительный анализ методов решения некорректных обратных задач для многоканальной гиперспектрометрии
}

\author{
() А.В. Гурылева, А.М. Хорохоров, В.И. Латышев \\ МГТУ им. Н.Э. Баумана, \\ 105005 Москва, Россия \\ e-mail: guryleva.av@gmail.com
}

Поступила в редакцию 03.06.2019 г.

В окончательной редакции 03.06.2019 г.

Принята к публикации 11.06.2019 г.

\begin{abstract}
Проведен сравнительный анализ вариаций осуществления многоканальной съемки объектов, проводимой с участием оптических фильтров и позволяющей значительно повысить спектральную разрешающую способность многоканальных гиперспектрометров с сохранением высокого пространственного разрешения. Также рассмотрены вычислительные возможности различных методов обработки данных многоканальной съемки. Показано, что восстановление спектральной плотности яркости по данным многоканальной съемки является некорректной задачей, а в качестве методов решения выбраны метод вейвлет-преобразования, метод регуляризации Тихонова и метод Годунова. Представлены результаты моделирования восстановления спектральной плотности яркости по данным многоканальной съемки с учетом наличия погрешностей в результатах измерений. Установлены границы применимости каждого метода. Метод регуляризации Тихонова предложен как наиболее устойчивый к погрешностям измерений. Проведено сравнение методов подбора оптических фильтров, участвующих в многоканальной съемке, относительно итоговой точности восстановления спектральной плотности яркости по результатам съемки и рекомендован классический метод. Определено оптимальное сочетание количества оптических фильтров и числа каналов съемки. Для широкого применения рекомендуются сочетания трех каналов с четырьмя оптическими фильтрами и восьми каналов с двумя оптическими фильтрами.
\end{abstract}

Ключевые слова: гиперспектрометрия, некорректные задачи, многоканальная съемка, метод регуляризации, оптические фильтры.

DOI: $10.21883 /$ OS.2019.10.48356.171-19

\section{Введение}

В работах [1] было показано, что применение метода с использованием многоканального спектрометра и одновременного введения оптических фильтров для измерения спектральной плотности яркости объекта в сочетании с применением методов решения обратных некорректных задач для обработки данных многоканальной съемки дает возможность значительно расширить возможности матричных приемников излучения с фильтрами Байера вплоть до их применения в гиперспектральной съемке без сканирования. При этом открытыми остаются вопросы выбора оптимального количества дополнительных оптических фильтров и их спектральных характеристик, а также способов извлечения гиперспектральной информации [2,3]. В настоящей работе эти вопросы рассматриваются с точки зрения эффективности и надежности практического использования.

Одним из основных препятствий к получению высокой повторяемости результатов восстановления спектральной плотности яркости по данным многоканальной съемки на практике является погрешность измерений сигнала приемника излучения [4,5]. Введение погрешности в моделирование метода многоканальной гиперспектрометрии позволит проверить надежность указанного метода и определить его место в современной науке и технике, в том числе привести математическое обоснование применения метода для получения гиперспектральных данных в практических приложениях [6,7].

В работе рассмотрены результаты моделирования восстановления спектральной плотности яркости объектов по косвенным эмпирическим измерениям сигналов матричным приемником излучения при различных условиях проведения съемки. Реконструкция спектральной плотности яркости проводилась различными численными методами, что позволило провести анализ их эффективности для описанной задачи и выявить наиболее применимый. Кроме того, рассматривались оптимальное число каналов съемки, количество оптических фильтров, участвующих в съемке по рассмотренному методу, вид кривой пропускания этих фильтров, а также комбинация этих параметров. Моделирование позволило составить требования к условиям проведения многоканальной съемки и к выбору метода обработки результатов съемки для достижения наибольшей точности восстановления спектра объекта.

\section{Моделирование и методы}

Процедура многоканальной съемки может быть модельно представлена в виде системы линейных инте- 
гральных уравнений, описывающих преобразование приемником излучения с фильтрами Байера спектральной плотности яркости объектов $L(\lambda)$ в набор сигналов $U$. Причем количество таких уравнений будет определяться количеством фильтров Байера $m$, обладающих спектральными чувствительностями $S(\lambda)$. При известном наборе значений результатов многоканальной $U$ и функций $S(\lambda)$ возможно определить с некоторой точностью исходную спектральную яркость объекта из решения описанной системы уравнений относительно $L(\lambda)$. Однако на практике для получения информации о функции $L(\lambda)$ с необходимым разрешением требуется привлечение дополнительной информации. С этой целью предлагается сделать несколько снимков через определенные оптические фильтры с известными спектральными функциями пропускания $\tau_{j}(\lambda),(j=1, \ldots, p)$. Если при съемке используется $p$ оптических фильтров, то количество уравнений для восстановления спектральной плотности яркости объектов увеличится на $m \times j$ аналогичных интегральных уравнений. Такую систему линейных интегральных уравнений допустимо заменить приближенным вычислением соответствующих интегралов с постоянным шагом $\Delta \lambda[1]$ :

$$
\left\{\begin{array}{l}
\sum_{i=1}^{n} L\left(\lambda_{i}\right) S_{B 1}\left(\lambda_{i}\right) v\left(\lambda_{i}\right) \tau_{j}\left(\lambda_{i}\right)=C U_{B 1 \tau j}, \\
\sum_{i=1}^{n} L\left(\lambda_{i}\right) S_{B 2}\left(\lambda_{i}\right) v\left(\lambda_{i}\right) \tau_{j}\left(\lambda_{i}\right)=C U_{B 2 \tau j}, j=0,1 \ldots p \\
\vdots \quad=\quad \vdots \\
\sum_{i=1}^{n} L\left(\lambda_{i}\right) S_{B m}\left(\lambda_{i}\right) v\left(\lambda_{i}\right) \tau_{j}\left(\lambda_{i}\right)=C U_{B m \tau j}
\end{array}\right.
$$

где $L\left(\lambda_{i}\right)$ - спектральная яркость объекта, $S_{B 1}\left(\lambda_{i}\right)$, $S_{B 2}\left(\lambda_{i}\right), S_{B m}\left(\lambda_{i}\right)$ - чувствительности пикселей для фильтров Байера матричного приемника излучения, $v\left(\lambda_{i}\right)-$ весовые коэффициенты шага, зависящие от способа численного интегрирования: методы прямоугольников, трапеций, Симпсона и т. П., $U_{B 1 \tau j}, U_{B 2 \tau j}, U_{B m \tau j}-$ величина сигнала пикселей, полученная при съемке объекта камерой с соответствующими фильтрами Байера через оптический фильтр с функцией пропускания $\tau_{j}\left(\lambda_{i}\right)$. (При $j=0$ следует принять $\tau_{j}\left(\lambda_{i}\right)=1$ - съемка объекта без оптического фильтра), $C-$ коэффициент, зависящий от неселективных потерь, $m$ - количество фильтров Байера, $n-$ количество точек разбиения по спектру, $i$ - номер шага разбиения, $i=1, \ldots, n$.

Система (1) может быть переписана в матричном виде, тогда спектральная плотность яркости будет определяться из решения следующего уравнения относительно l:

$$
S \mathbf{l}=\mathbf{u},
$$

где $\mathbf{I}$ - вектор из $n$ элементов, определяющий спектральную плотность яркости объекта, $S$ - матрица размера $(m(p+1)) \times n$, учитывающая чувствительности пикселей для фильтров Байера матричного приемника излучения, известные спектральные функции пропускания $\tau_{j}\left(\lambda_{i}\right)$ и весовые коэффициенты шага $v\left(\lambda_{i}\right), \mathbf{u}-$ вектор из $m(p+1)$ элементов, определяющий величины сигналов пикселей, полученные при съемке объекта камерой с фильтрами Байера и оптическими фильтрами с учетом погрешности.

В настоящей работе приведены результаты численного моделирования описанного метода восстановления спектральной плотности яркости объекта по результатам многоканальной съемки. Результатами первого этапа моделирования были величины сигналов пикселей матричного приемника излучения, определенные по известным чувствительностям матричного приемника с фильтрами Байера $S_{B m}\left(\lambda_{i}\right)$, известной спектральной плотности яркости объекта $L\left(\lambda_{i}\right)$ и известным спектральным функциям пропускания $\tau_{j}(\lambda)$.

Для приближения моделирования к реальным измерениям считалось, что восстановление функции $L(\lambda)$ проводилось в ситуации, когда значения сигналов на пикселях $U_{B m} \tau_{j}$ измеряются с некоторой погрешностью [8-10]. С этой целью в величины, определенные на предыдушем этапе, вводилась погрешность $\delta$. Погрешность генерировалась случайным образом и имела значения $\delta=0.5,1.0,2.5 \%$ от максимального значения сигнала. Далее по искаженному таким образом сигналу определялась с некоторой точностью спектральная яркость $L^{*}\left(\lambda_{i}\right)$ из выражения (2). Мерой соответствия восстановленного таким образом спектра исходному являлась относительная погрешность, выраженная в процентах.

Решение системы уравнений (1) относительно $L(\lambda)$, представляющее собой некорректно поставленную задачу, проводилось соответствующими методами $[1,11,12]$. В работе рассматриваются метод Гревиля, метод вейвлет-преобразования, метод регуляризации Тихонова и метод Годунова [13-16], приводятся результаты сравнения устойчивости каждого метода к погрешности в исходных данных. Подробное описание используемых в настоящей работе методов дано в работах $[1,4,13-16]$.

Подбор оптимального набора оптических фильтров представляется одним из способов увеличения точности описанной методики мультиспектральной съемки. В литературе [17-21] описан ряд методов для определения наилучшего набора оптических фильтров при решении задач, аналогичных задаче настоящей работы.

Эмпирический метод. Метод заключается в том, что рассматривается совокупность кривых пропускания, образованных $k$ комбинациями дополнительных оптических фильтров с фильтрами Байера. При этом комбинации выбираются так, чтобы максимумы их кривых пропускания были равномерно распределены по всему рабочему диапазону длин волн $[18,21]$. На рис. 1, $a$ показаны кривые пропускания тех комбинаций фильтров из оптических стекол ЖЗС-5, ЖЗС-18, С $3 \mathrm{C}-16$ и СС-1 с фильтрами Байера стандартной RGB-матрицы, которые представляются наиболее рациональными (всего $k=9$ ) и использованы в дальнейшем моделировании.

Метод снижсения линейной зависимости строк матрицы (метод снижения обусловленности). По мнению авторов метода [17] наилучшие результаты с точки зрения снижения степени обусловленности матрицы $S$ можно обеспечить оптическими фильтрами, 

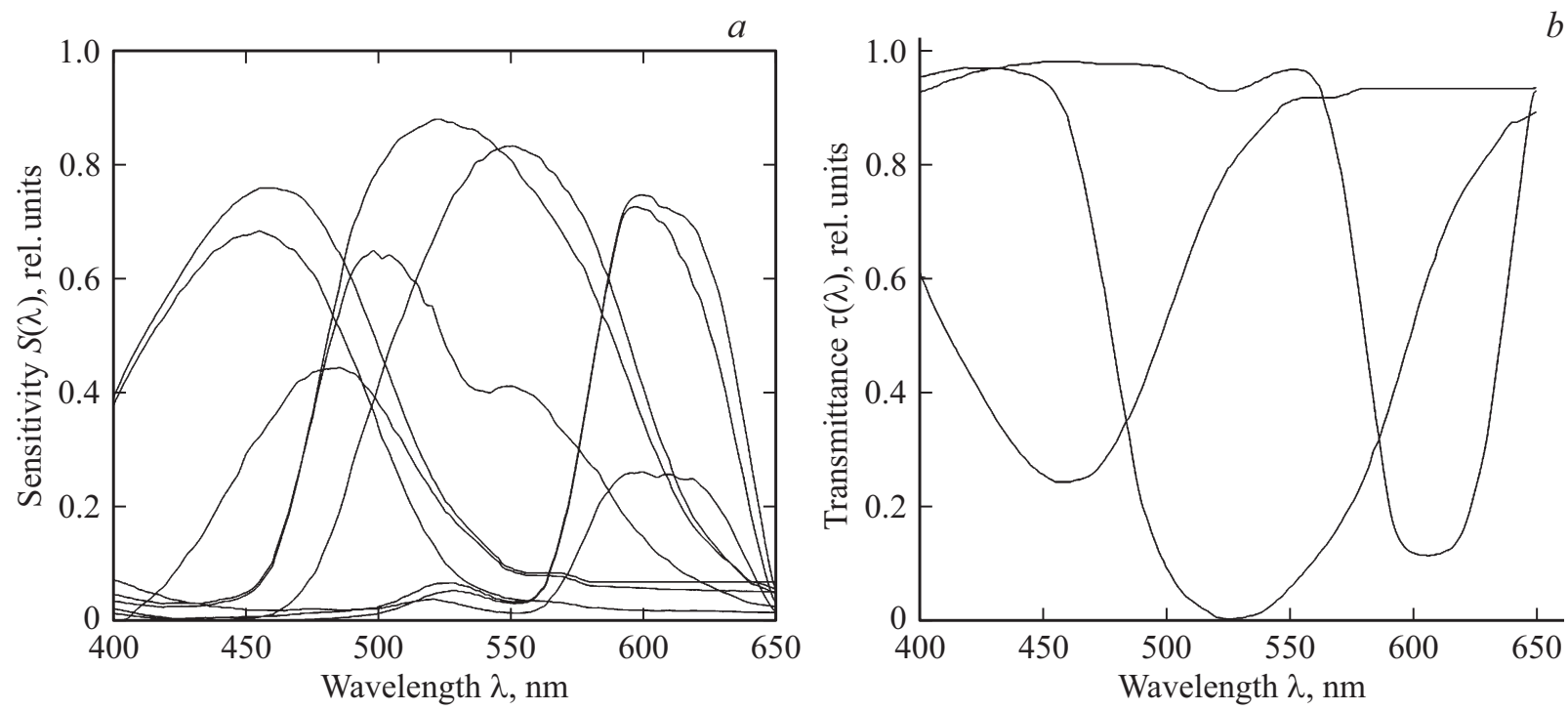

Рис. 1. Графики функций чувствительностей набора комбинаций оптических фильтров с фильтрами Байера, полученных эмпирическим методом $(a)$, кривые пропускания оптических фильтров, обратных функциям чувствительности фильтров Байера $(b)$.

функции пропускания которых определяется выражением $\tau_{j}\left(\lambda_{i}\right)=1-S_{f B m}\left(\lambda_{i}\right)$. Итоговые используемые кривые пропускания оптических фильтров для стандартной RGB-матрицы приведены на рис. $1, b$.

Большинство расчетов в настоящей работе выполнено с применением классического метода, заключающегося в подборе фильтров с максимально различным видом кривых пропускания и равномерным покрытием всего рабочего диапазона длин волн $[17,18]$. В качестве таких фильтров были использованы фильтры, выполненные из оптических стекол ЖЗС-5, ЖЗС-18, С3С-16 и СС-1.

Следует отметить, что в работе авторами предлагается сравнивать методы при равном количестве комбинаций $k$ оптических фильтров с фильтрами Байера, а именно $k=9$. При этом количество кадров, необходимых для формирования таких комбинаций, в каждом методе будет разным и будет определяться числом используемых фильтров $p$. Для получения 9 комбинаций эмпирическим методом требуется сделать 4 кадра $(p=4)$, для метода снижения обусловленности $p=3$, для классического метода $p=2$.

Кроме того, в работе промоделированы измерения, проведенные с использованием разного количества оптических фильтров и фильтров Байера. Ожидалось, что с увеличением их количества точность измерений будет увеличиваться [22,23], однако требовалось определить достаточное число фильтров для требуемой точности [18].

Моделирование проводилось на трех эталонных объектах [1] с различной спектральной плотностью яркости, определенной спектрофотометром Perkin Elmer Lambda 950. Графики спектральной яркости объектов представлены на рис. 2 сплошными линиями без выделения или с выделением кружками или звездочками.

Рассматривались два матричных приемника излучения с различным числом фильтров Байера: $m=3$ (филь- тры RGB) и $m=8$ (полосовые фильтры). Для единообразия представления результатов проведенных расчетов моделирование восстановления исходной спектральной плотности яркости перечисленными методами в число включаемых поочередно в ход лучей оптических фильтров для матрицы с $m=3$ вошли фильтры, выполненные из оптического стекла ЖЗС-5, ЖЗС-18, СЗС-16 и CC-1 (всего $p=4)$, для матрицы с $m=8$ применялись ЖЗС-18 и СС-1 (всего $p=2)$.Также определено влияние метода выбора наиболее подходящих оптических стекол для мультиспектральных измерений и выявлена оптимальная комбинация числа фильтров Байера $m$ и количества оптических стекол $p$. Кроме того, все расчеты в работе приведены для различных величин погрешности, внесенной случайным образом в сигнал с матричного приемника излучения: $\delta=0.5,1.0,2.5 \%$ от максимального значения сигнала.

\section{Результаты и обсуждение}

В табл. 1 представлены усредненные величины относительной погрешности восстановления спектральной плотности яркости по трем объектам, определенной методами решения некорректных обратных задач, описанными выше.

Исходя из данных табл. 1 можно отметить следующее. Метод Гревиля дает удовлетворительные результаты при отсутствии погрешности в регистрируемом сигнале [1], однако при ее наличии относительная погрешность восстановления резко возрастает на порядки, что не позволяет рекомендовать его для практического применения. Метод вейвлет-преобразования показал большую устойчивость, однако восстановление с его помощью спектра яркости по исходным данным с погрешностью, составляющей более $0.5 \%$ для $m=8$, 
Таблица 1. Результаты моделирования восстановления спектральной плотности яркости по данным многоканальной съемки методами решения некорректных задач

\begin{tabular}{|c|c|c|c|c|c|c|c|c|}
\hline \multirow{3}{*}{$\begin{array}{c}\text { Величина } \\
\text { погрешности } \\
\text { измерения } \\
\text { сигнала, \% }\end{array}$} & \multicolumn{8}{|c|}{ Относительная погрешность, \% } \\
\hline & \multicolumn{2}{|c|}{ Метод Гревиля } & \multicolumn{2}{|c|}{$\begin{array}{l}\text { Метод вейвлет- } \\
\text { преобразования }\end{array}$} & \multicolumn{2}{|c|}{$\begin{array}{c}\text { Метод регуляризации } \\
\text { Тихонова }\end{array}$} & \multicolumn{2}{|c|}{ Метод Годунова } \\
\hline & $\begin{aligned} m & =3 \\
p & =4\end{aligned}$ & $\begin{aligned} m & =8 \\
p & =2\end{aligned}$ & $\begin{aligned} m & =3 \\
p & =4\end{aligned}$ & $\begin{aligned} m & =8 \\
p & =2\end{aligned}$ & $\begin{aligned} m & =3 \\
p & =4\end{aligned}$ & $\begin{aligned} m & =8 \\
p & =2\end{aligned}$ & $\begin{aligned} m & =3 \\
p & =4\end{aligned}$ & $\begin{aligned} m & =8 \\
p & =2\end{aligned}$ \\
\hline 0.5 & $>100$ & $>100$ & $>100$ & 1.19 & 2.01 & 1.02 & 2.67 & 2.27 \\
\hline 1.0 & $>100$ & $>100$ & $>100$ & 50 & 3.28 & 1.27 & 4.58 & 2.69 \\
\hline 2.5 & $>100$ & $>100$ & $>100$ & $>100$ & 4.32 & 2.67 & 6.47 & 4.25 \\
\hline
\end{tabular}

$p=2$, не представляется возможным. Метод Годунова хорошо справляется с вычислениями при погрешности измерения сигнала $\delta=0.5 \%$ и дает удовлетворительные результаты при погрешности $\delta=1.0 \%$. Наиболее устойчивые результаты по отношению к погрешности в исходных данных показывает метод регуляризации Тихонова. Усредненная относительная погрешность для метода Тихонова при $\delta=2.5 \%$ не более $4.3 \%$ при $m=3, p=4$ и $1.8 \%$ при $m=8, p=2$, что позволяет определять исходный спектр объекта с погрешностью, не превышающей погрешность прибора, которым проводится регистрация сигнала в плоскости изображений при $m=8, p=2$.

Выбор оптимального метода реконструкции спектральной плотности яркости должен производиться в соответствии с классом точности измерительного оборудования и требуемой точности вычисления, однако авторами для установленной цели рекомендуется метод регуляризации Тихонова как наиболее универсальный. Формирование дальнейших вычислений для оценки различных вариаций исходных данных многоканальной съемки также проводилось с помощью метода регуляризации Тихонова ввиду его универсальности, однако ожидается, что и для других методов восстановления спектральной плотности яркости выводы, сделанные ниже, окажутся верными.

Точность восстановления исходной спектральной плотности яркости объектов при наличии определенных погрешностей представляется недостаточной для практического применения, поэтому графическая интерпретация результатов работы была произведена только для тех реконструированных кривых, которые несут полезную информационную нагрузку. На рис. $2, a-f$ пунктирными линиями проиллюстрированы результаты восстановления исходной спектральной плотности яркости, проведенного четырьмя методами при различных значениях погрешности $\delta$ для двух сочетаний количества фильтров Байера $m$ и числа оптических фильтров $p$ $(m=3, p=4$ и $m=8, p=2)$.

В ходе работе были проверены три метода подбора комбинаций $k$ оптических фильтров с фильтрами Байера. В табл. 2 собраны значения относительных погрешностей восстановления спектральной плотности яркости для погрешностей регистрации сигнала $\delta=0.5,1.0,2.5 \%$, полученные с применением этих методов.

Данные табл. 2 показывают, что использование эмпирического метода при съемке дает наибольшую относительную погрешность восстановления спектра яркости. Метод снижения обусловленности выступает как метод, дающий наименьшую относительную погрешность восстановления. Однако последний требует создания или подбора фильтров, отвечающих определенной функции, меняющейся в зависимости от матричного приемника излучения, что представляется наиболее подходящим лишь в отдельных случаях. Также следует отметить, что для получения равного количества комбинаций $k$ в каждом методе нужно было сделать разное количество кадров по числу фильтров $p$, и в классическом методе соотношение количества кадров относительно количества используемых комбинаций наиболее выгодное. Кроме того, показано, что при использовании одинакового количества фильтров для метода снижения обусловленности и классического метода последний имеет меньшую относительную погрешность. Таким образом, классический метод рекомендуется авторами как более универсальный.

В табл. 3 приведены значения усредненной относительной погрешности восстановления спектральной плотности яркости методом регуляризации Тихонова для разных сочетаний числа оптических фильтров и числа фильтров Байера.

В результате моделирования определено, что оптимальными сочетаниями по соотношению количества кадров в съемке для одного измерения спектральной плотности яркости образца, сложности производимых для решения уравнения (3) вычислений и итоговой относительной погрешности восстановления спектра являются сочетания стандартной трехканальной матрицы с четырьмя оптическими фильтрами и восьмиканальной матрицы с двумя оптическими фильтрами. Следует также отметить, что усредненная по трем образцам относительная погрешность реконструкции спектра по данным съемки с сочетанием восьмиканальной матрицы и одного оптического фильтра является также удовлетворительной, однако от образца к образцу имеет значительные различия. 

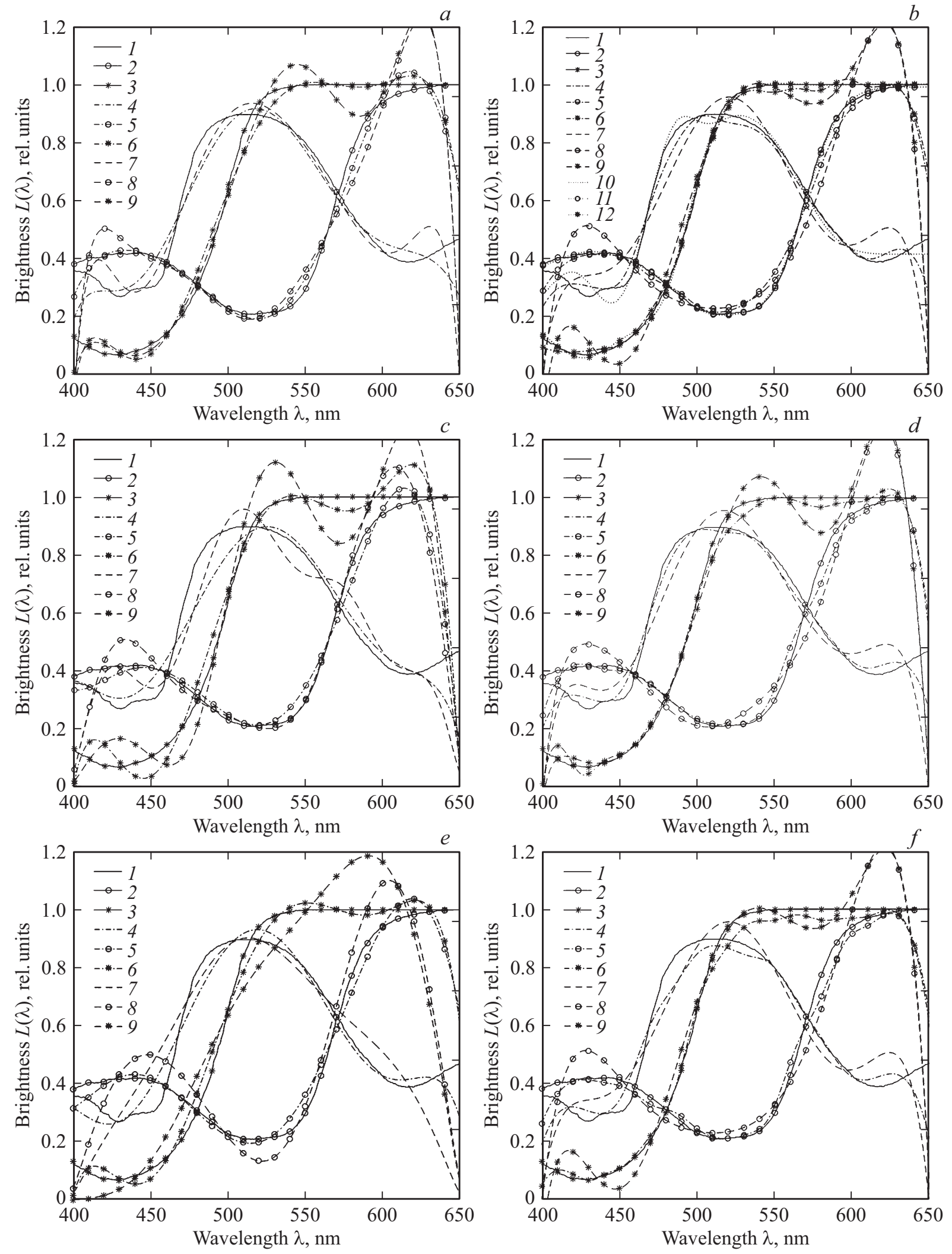

Рис. 2. Кривые исходной спектральной плотности яркости и спектральной плотности яркости, восстановленной при различных условиях моделирования: $m=3, p=4, \delta=0.5 \%(a) ; m=8, p=2, \delta=0.5 \%(b) ; m=3, p=4, \delta=1.0 \%(c) ; m=8, p=2$, $\delta=1.0 \%(d) ; m=3, p=4, \delta=2.5 \%(e) ; m=8, p=2, \delta=2.5 \%(f)$. Исходные образцы - $1,2,3$; кривые, восстановленные методом регуляризации Тихонова - 4, 5; кривые, восстановленные методом Годунова - 7, 8, 9; кривые, восстановленные методом вейвлет-преобразования - 10, 11, 12 . 
Таблица 2. Результаты моделирования восстановления спектральной плотности яркости при различных комбинациях оптических фильтров с фильтрами Байера

\begin{tabular}{|c|c|c|c|c|}
\hline \multirow{4}{*}{$\begin{array}{c}\text { Величина } \\
\text { погрешности } \\
\text { измерения } \\
\text { сигнала, \% }\end{array}$} & \multicolumn{4}{|c|}{ Относительная погрешность,\% } \\
\hline & \multicolumn{4}{|c|}{ Метод регуляризации Тихонова } \\
\hline & \multirow{2}{*}{$\begin{array}{c}\text { Эмпирический } \\
\text { метод }\end{array}$} & \multirow{2}{*}{$\begin{array}{c}\text { Метод снижения } \\
\text { обусловленности }\end{array}$} & \multicolumn{2}{|c|}{ Классический метод } \\
\hline & & & $\begin{array}{c}m=3 \\
k=9, p=2\end{array}$ & $\begin{array}{c}m=3 \\
k=12, p=3\end{array}$ \\
\hline 0.5 & 3.90 & 3.15 & 3.55 & 2.65 \\
\hline 1.0 & 5.55 & 4.07 & 5.01 & 4.02 \\
\hline 2.5 & 9.75 & 5.96 & 6.51 & 5.70 \\
\hline
\end{tabular}

Таблица 3. Результаты моделирования восстановления спектральной плотности яркости при разном количестве кадров, участвующих в обработке

\begin{tabular}{c|c|c|c|c|c|c}
\hline \multirow{2}{*}{$\begin{array}{c}\text { Величина } \\
\text { погрешности }\end{array}$} & \multicolumn{5}{|c}{ Относительная погрешность, $\%$} \\
\cline { 2 - 7 } измерения & \multicolumn{5}{|c}{ Метод регуляризации Тихонова } \\
\cline { 2 - 7 } сигнала, \% & $m=3$ & $m=8$ & $m=3$ & $m=8$ & $m=3$ & $m=8$ \\
& $p=3$ & $p=1$ & $p=4$ & $p=2$ & $p=5$ & $p=4$ \\
\hline 0.5 & 2.65 & 1.45 & 2.01 & 1.02 & 1.97 & 1.10 \\
1.0 & 4.02 & 2.03 & 3.28 & 1.27 & 3.25 & 1.15 \\
2.5 & 5.70 & 3.42 & 4.32 & 2.67 & 4.30 & 2.60
\end{tabular}

\section{Выводы}

В работе представлены результаты сравнительного анализа вариаций проведения многоканальной съемки объектов гиперспектрометром с высоким значением пространственной и спектральной разрешающей способности, достигаемой получением дополнительной информации о спектральных характеристиках объекта за счет его сьемки через специальные оптические фильтры; также рассмотрены вычислительные возможности различных методов обработки данных многоканальной съемки.

Выявлено, что при небольших погрешностях измерений (порядка $0.5 \%$ от максимального значения сигнала) наименьшую относительную погрешность восстановления спектральной плотности яркости показывает метод вейвлет-преобразований. С повышением погрешности измерений до $1 \%$ наиболее предпочтительными становятся методы регуляризации Тихонова и метод Годунова. При больших погрешностях исходных данных только метод регуляризации Тихонова позволяет получить результаты не хуже класса точности, используемой для съемки аппаратуры, и поэтому авторами рекомендуется метод Тихонова как наиболее универсальный.

Среди методов подбора оптических фильтров можно выделить классический метод, с применением которого была проведена большая часть вычислений работы, и метод снижения обусловленности. Последний показывает лучшие результаты, но требует создания или подбора фильтров, отвечающих определенной функции, зависящей от используемого при съемке матричного приемника излучения, что в отдельных случаях представляется возможным и оправданным, однако для большинства задач авторы предлагают классический метод ввиду его универсальности.

Также в ходе работы было выбрано оптимальное сочетание количества оптических фильтров, применяемых в съемке, и числа фильтров Байера матричного приемника излучения. Исходя из технологичности решения и величины погрешности восстановления спектральной плотности яркости для широкого применения рекомендуются сочетания стандартной трехканальной матрицы с четырьмя оптическими фильтрами и восьмиканальной матрицы с двумя оптическими фильтрами.

В целом проведенное исследование подтверждает возможность применения многоканальной съемки для гиперспектрометрии обсуждаемыми методами в практических целях ввиду устойчиво хороших результатов моделирования их работы. Кроме того, работа дает расширенную информацию о требованиях к исходным данным проведения обсуждаемой многоканальной съемки. Предметом дальнейших исследований может явиться определение других типов базиса вейвлет-преобразования, а также проведение физического эксперимента по установлению возможностей многоканальной съемки для гиперспектрометрии. 


\section{Список литературы}

[1] Хорохоров А.М., Введенская А.В., Ширанков А.Ф., Кобозев В.С. // Прикладная оптика 2018. СПб.: Оптическое общество им. Д.С. Рождественского, 2018.

[2] Connah D., Alsam A., Hardeberg J.Y. // J. Imaging Science and Technology. 2006. V. 50. N 1. P. 45. doi 10.2352/j.imagingsci.technol.

[3] Trukhanov S.V. // Proceeding of the XX-th International Open Science Conference „Modern informatization problems in economics and safety". USA, Yelm: Science Book Publishing House LLC, 2015. P. 56.

[4] Морозов В.А. // Выч. мет. программирование. 2003. Т. 4. B. 1. C. 130.

[5] Zhao Y., Berns R.S. // Color Res. Appl. 2007. V. 32. P. 343.

[6] Ахметов Р.Н., Стратилатов Н.Р., Юдаков А.А., Везенов В.И., Еремеев В.В. // Исследование Земли из космоса. 2014. № 1. С. 17.

[7] Chlebda D.K., Rogulska A., Łojewski T. // Spectrochimica Acta Part A: Molecular and Biomolecular Spectroscopy. 2017. V. 185. P. 55. doi 10.1016/j.saa.2017.05.037

[8] Арапов С.Ю., Арапова С.П., Дубинин И.С., Сергеев А.П. Восстановление спектров отражения тестовых полей по данным мультиспектральной фотосъемки. Екатеринбург: УрФУ, 2015. С. 21.

[9] Федотов А.М. Некорректные задачи со случайными ошибками в данных. Новосибирск: Наука, 1990. С. 280.

[10] Brill M.H. // Color Res. Appl. 2002. V. 27. N 4. P. 304. doi 10.1002/col.10073

[11] Valero E.M., Hu Y., Hernández-Andrés J., Eckhard T., Nieves J.L., Romero J., Schnitzlein M., Nowack D. // Color Res. Appl. 2012. V. 39. P. 1.

[12] Vogel C.R. Computational Methods for Inverse Problems. SIAM, Philadelphia, 2002. P. 179.

[13] El-Rifai I. // Int. J. Image Process. 2013. V. 7. N 3. P. 278.

[14] Новиков Л.В. // Научное приборостроение. 2000. Т. 10. № 3. C. 70.

[15] Тихонов А.Н., Арсенин В.Я. Методы решения некорректных задач. М.: Наука, 1986. С. 288.

[16] Годунов С.К., Антонов А.Г., Кирилюк О.П., Костин В.И. Гарантированная точность решения систем линейных уравнений в евклидовых. Новосибирск: Наука, 1988. C. 456.

[17] Hardeberg J.Y. Filter Selection for Multispectral Color Image Acquisition IS\&T'sPICS Conference. USA, Rochester, NY. 2003. V. 48. N 2. P. 177.

[18] Connah D., Alsam A., Hardeberg J.Y. // J. Imaging Science and Technology. 2006. V. 50 (1). P. 45.

[19] Alejandro Ribés, Francis Schmitt, Hans Brettel. // Proceedings of 3rd International Conference on Multispectral Color Science. Joensuu, Finland. 2001. P. 19.

[20] Darling B.A., Ferwerda J.A., oy Berns R.S., Chen T. // Color and Imaging Conference: 19th Color and Imaging Conference. Final Program and Proceedings. San Jose, California. 2011. P. 345.

[21] Арапов С.Ю., Тарасов Д.А., Сергеев А.П., Колмогоров Ю.Н. // Изв. вузов. Проблемы полиграфии и издательского дела. 2012. № 6. С. 17.
[22] Masahiro Y., Hideaki H., Nagaaki O. // J. Imaging Sci. Technol. 2008. V. 52 (1). doi 10.2352/J.ImagingSci.Technol

[23] Burns P.D., Berns R.S. // Color Imaging Conf. 1996. V. 1996. N 1. P. 19. 